\title{
A scalable pipeline for local ancestry inference using tens of thousands of reference haplotypes
}

\author{
Eric Y. Durand*, Chuong B. Do*, Peter R. Wilton*‡, Joanna L. Mountain, Adam Auton, \\ G. David Poznik ${ }^{\dagger \ddagger}$, J. Michael Macpherson ${ }^{\dagger}$ \\ 23andMe, Inc., Mountain View, CA, USA \\ ${ }^{* \dagger}$ Authors contributed equally \\ ¥To whom correspondence should be addressed: \{peterw,dpoznik\}@23andme.com
}

Original: October 17, 2014

Updated: December 7, 2020

\begin{abstract}
Ancestry deconvolution is the task of identifying the ancestral origins of chromosomal segments of admixed individuals. It has important applications, from mapping disease genes to identifying loci potentially under natural selection. However, most existing methods are limited to a small number of ancestral populations and are unsuitable for large-scale applications.

In this article, we describe Ancestry Composition, a modular pipeline for accurate and efficient ancestry deconvolution. In the first stage, a string-kernel support-vector-machines classifier assigns provisional ancestry labels to short statistically phased genomic segments. In the second stage, an autoregressive pair hidden Markov model corrects phasing errors, smooths local ancestry estimates, and computes confidence scores.

Using publicly available datasets and more than 12,000 individuals from the customer database of the personal genetics company, 23 andMe, Inc., we have constructed a reference panel containing more than 14,000 unrelated individuals of unadmixed ancestry. We used principal components analysis (PCA) and uniform manifold approximation and projection (UMAP) to identify genetic clusters and define 45 distinct reference populations upon which to train our method. In cross-validation experiments, Ancestry Composition achieves high precision and recall.
\end{abstract}

\section{Introduction}

An individual's genome can be viewed as a mosaic of chromosomal segments of potentially different ancestries (Falush et al., 2003; Tang et al., 2005). Ancestry deconvolution, or local ancestry inference (LAI), is the task of resolving the ancestral origin of such segments. Robust ancestry deconvolution enables several important lines of research, including admixture-based mapping of disease genes (Seldin et al., 2011); disease-association studies, in which controlling for population structure is essential (Price et al., 2010); and studies of population history (Novembre and Ramachandran, 2011; Hellenthal et al., 2014).

As LAI is a classification problem, there are two general approaches one may take. One approach is generative, in which one models the joint probability of haplotypes and ancestries, and the other is discriminative, in which one models the conditional probability of ancestries given haplotype data (see section 4 for a fuller discussion of this distinction). Several ancestry-deconvolution methods implement generative models in which the ancestry of chromosomal segments is inferred using hidden Markov models (e.g., Tang et al., 2006; Price et al., 2009). Other generative approaches have used sliding-window algorithms (e.g. Pasaniuc et al., 2009). In contrast to generative approaches, discriminative approaches do not attempt to fully model the underlying admixture process. Instead, they attempt to learn directly from segments of known ancestry the conditional distribution of ancestries given haplotype data. Discriminative models make fewer assumptions about the demographic process underlying admixture and typically scale better to large datasets (Omberg et al., 2012; Kumar et al., 2020). A number of discriminative approaches have been described (Brisbin et al., 2012; Omberg et al., 2012; Maples et al., 2013; Kumar et al., 2020; Montserrat et al., 2020).

Previous LAI methods have also differed in how they treat chromosome phase. Some early methods relied on unphased 
genotype data and predicted for each genetic marker whether zero, one, or two alleles derive from a specified ancestral population (e.g., Pasaniuc et al., 2009; Sundquist et al., 2008; Price et al., 2009). However, there is much information to be gleaned from phase. An individual may inherit very different ancestries from their mother and father, and the ability to represent and model these differences provides greater power for identifying ancestries and enables parental contributions to be distinguished. Some methods incorporate phase information by prephasing genotypes (Brisbin et al., 2012; Maples et al., 2013). Others phase the input genotypes as part of the analysis (Bercovici et al., 2012). However, even with the availability of population-scale genetic datasets (e.g., Bycroft et al., 2018; McCarthy et al., 2016) and breakthroughs in statistical phasing methodology (e.g., Loh et al., 2016), it remains challenging to recover chromosome-scale phase information. Thus, it is beneficial to model phase errors as part of the data generation process.

In this article, we present an update to Ancestry Composition, a modular two-stage LAI pipeline originally described in Durand et al. (2014). In a manner similar in spirit to (Omberg et al., 2012; Maples et al., 2013), Ancestry Composition uses a discriminative approach to determine the ancestral origins of short chromosomal segments. It subsequently corrects these assignments with a generative model that jointly models the true ancestries of each haplotype, correlations in local assignments, and errors in haplotype phase inference. We have trained Ancestry-Composition models using a reference panel of more than 14,000 individuals with known ancestry, most of whom are customers of 23andMe, Inc., a personal genomics company. Ancestry Composition achieves high precision and recall when labeling chromosomal segments from more than 45 worldwide populations. In contrast, most existing LAI methods tend to be limited to a few ancestral populations and typically lack power to distinguish between closely related populations (Pasaniuc et al., 2009). We designed the method to function in a online setting in which an analyst or consumer product must continuously predict new individuals. As such, Ancestry Composition scales linearly with the number of individuals to analyze.

\section{Methods}

Ancestry Composition consists of two largely independent modules:

1. A local classifier module, wherein a string-kernel support-vector-machines classifier (SKSVM) assigns provisional ancestry labels to short locally phased genomic regions.

2. An error-correction module, wherein an autoregressive pair hidden Markov model (APHMM) corrects phasing errors, smooths provisional ancestry estimates, and assigns confidence scores.

\subsection{Local classifier}

The task of the local classifier is to assign each marker along each haplotype to one of $K$ reference populations. The local classifier starts by splitting each haplotype into $S$ windows of $M$ biallelic markers. Each window is treated independently and is assumed to have a single ancestral origin. Thus, for each haplotype, the local classifier returns a vector $c_{1: S}$, where $c_{i} \in\{1 \ldots K\}$ is the hard-clustering value assigned to window $i$. We implemented the local classifier using string-kernel support vector machines, a discriminative classifier.

\subsubsection{String-kernel support vector machine}

Support Vector Machines (SVMs) are a class of supervised learning algorithms first introduced by Vapnik (1998). In its most basic form, an SVM is a non-probabilistic binary linear classifier. That is, it learns a linear decision boundary that can be used to discriminate between two classes. SVMs can be extended to problems that are not linearly separable using the soft-margin technique. For more details, see Cristianini and Shawe-Taylor (2000).

Consider a set of training data $\left\{\left(\mathbf{x}_{i}, y_{i}\right)\right\}_{1: N}$, where for each $i, \mathbf{x}_{i}$ is a feature vector in $\mathbb{R}^{d}$ and $y_{i} \in\{-1,1\}$ is a class label. The SVM learns the decision boundary by solving the following quadratic programming optimization problem: 


$$
\begin{array}{lll}
\min _{\mathbf{w} \in \mathbb{R}^{d}, \boldsymbol{\xi} \in \mathbb{R}^{N}, b \in \mathbb{R}} & \frac{1}{2}\|\mathbf{w}\|^{2}+C \sum_{i=1}^{N} \xi_{i} \\
\text { subject to } & \begin{cases}y_{i}\left(\mathbf{w}^{T} \mathbf{x}_{i}-b\right) \geq 1-\xi_{i} & \forall i . \\
\xi_{i} \geq 0 & \end{cases}
\end{array}
$$

$C$ is a tuning parameter that, in practice, we generally set to 1 .

Encoding the feature vectors In our application, each feature vector $\mathbf{x}_{i}$ represents the encoding of a haplotype window of $M$ biallelic markers from a prephased haplotype. One natural encoding is to use one feature per marker, with each feature encoding the presence or absence of the minor allele. However, this encoding fails to capture the the spatial relationship of consecutive markers within the window (i.e., the linkage pattern), a distinguishing feature of genetic variation. Instead, we use every possible $k$-mer $(k \in\{1 \ldots M\})$ as our features. For a haplotype segment of $M$ biallelic markers, there are $d=\sum_{k=1}^{M}(M-k+1) 2^{k}$ possible $k$-mers. When $M=100, d$ is on the order of $10^{30}$, so it is not feasible to directly construct feature vectors with this many dimensions. In the next section, we introduce a string kernel that enables working with our high-dimensional feature set.

String kernel A key property of SVMs is that solving (1) is equivalent to solving the following dual quadratic programming problem:

$$
\begin{aligned}
\max _{\alpha \in \mathbb{R}^{N}} & \sum_{i=1}^{N} \alpha_{i}-\frac{1}{2} \sum_{i, j=1}^{N} \alpha_{i} \alpha_{j} y_{i} y_{j} \mathbf{x}_{i}^{T} \mathbf{x}_{j} \\
\text { subject to } & \left\{\begin{array}{ll}
0 \leq \alpha_{i} \leq C \\
\sum_{i=1}^{N} \alpha_{i} y_{i}=0
\end{array} \quad \forall i .\right.
\end{aligned}
$$

The dual representation of the SVM optimization problem depends only on the inner product $\mathbf{x}_{i}^{T} \mathbf{x}_{j}$, which means we can introduce kernels (Boser et al., 1992). Kernels provide a way to map observations to a high-dimensional feature space, thereby offering an enormous computational advantage, as they can be evaluated without explicitly calculating feature vectors. Denoting the input space as $\chi$, let $\phi: \chi \rightarrow\{0,1\}^{d}$ be the mapping such that for any segment $x$ of length $M, \phi(x)$ is the vector whose elements denote the presence or absence of each of the $d$ possible $k$-mers in $x$. We define our string kernel as, $\forall i, j \in\{1, \ldots, N\}$,

$$
\begin{aligned}
K\left(x_{i}, x_{j}\right) & =\phi\left(x_{i}\right)^{T} \phi\left(x_{j}\right) \\
& =\sum_{k=1}^{M} \sum_{l=1}^{M-k+1} \mathbb{1}\left\{u_{k l i}=u_{k l j}\right\}
\end{aligned}
$$

where $u_{k l i}$ is the $k$-mer starting at position $l$ in haplotype window $i$. Our kernel is a special case of the weighted degree kernel (Rätsch et al., 2006). Standard dynamic programming techniques can be used to evaluate $K\left(x_{i}, x_{j}\right)$ in $O(M)$ operations without explicitly enumerating the $d$ features for each mapped input vector. Thus, the string kernel enables us to extract a large amount of information from each haplotype window.

Multiclass SVMs SVMs are fundamentally binary classifiers, but in this setting we are concerned with deciding among 45 possible populations. To assign a single hard-clustering value $k \in\{1, \ldots, K\}$ to a haplotype window, we trained $\left(\begin{array}{c}K \\ 2\end{array}\right)$ classifiers, one for each pair of populations. We assign each haplotype segment to a single population using a straightforward majority vote across all pairs. We also experimented with a one-vs-all approach that did not perform as well.

\subsubsection{Training data}

We trained the local classifier on $\sim 14,400$ unrelated individuals, each with unadmixed ancestry from one of $K=45$ reference populations (Table 1). This reference panel includes $\sim 11,800$ research-consented 23andMe customers, 600 
individuals from non-customer 23andMe datasets, and 2000 individuals from publicly available datasets, including the 1000 Genomes Project (The 1000 Genomes Project Consortium, 2015) and the Human Genome Diversity Panel (Cann et al., 2002).

To ensure that all the reference individuals were distantly related, we used the method described in Henn et al. (2012) to estimate identity-by-descent (IBD) sharing between each pair of individuals and removed individuals from the sample until no pair shared more than an $100 \mathrm{cM}$. We then conducted principal components analysis (PCA) and uniform manifold approximation and projection (UMAP; McInnes et al., 2018; Diaz-Papkovich et al., 2019) to identify population structure, which, when paired with survey data and analyzed jointly with the well-curated external reference panels, enabled us to define our 45 reference populations and flag outliers for removal.

For most reference populations, the research-consented 23andMe customers reported in survey responses that their four grandparents were born in a single country. For regions with large multiethnic countries (e.g., South Asia), we also required that an individual's four grandparents either spoke a single regional language or were born in one state. Free-text responses on grandparental national, ethnic, religious, or other identities enabled us to construct reference panels for populations not defined by specific geographic regions (e.g., Ashkenazi Jews).

\subsubsection{Window size}

A key assumption of the local classifier is that the haplotype segment within each window derives from a single population. Thus, the window-size parameter influences the timing of the admixture we can address. For example, if we sought only to infer "local" admixture in first-generation admixed individuals, then windows could potentially span entire chromosomes. More generally, if we assume a simple admixture model in which two reference populations mixed $T$ generations ago, then the expected length of a single-ancestry segment is $100 /(2 T) \mathrm{cM}$.

Phasing switch errors also limit the sizes of segments we can consider. If a switch error occurs within a haplotype window, our assumption that the haplotype segment covered by the window has a single ancestry may no longer be valid. Thus, it is necessary to choose a window size small enough to ensure that most windows are free of switch errors. On the other hand, longer windows contain more information, which increases the power of the SKSVM to separate reference populations.

For the analyses presented in this article, we used a window size of 300 markers. This corresponds to $\sim 0.6 \mathrm{cM}$ per window and, on a genotyping platform measuring $~ 540,000$ markers, divides the genome into $\sim 1800$ windows. We chose this window size because we find it provides a good balance between retaining ancestry-related information within windows and precluding recombination events and phasing errors within them.

\subsection{Error-correction module}

The local classifier generates noisy ancestry estimates, so we developed an error-correction module to smooth hardclustering assignments using information from adjacent windows. To compute smoothed assignment probabilities, we have implemented an autoregressive pair hidden Markov model (APHMM) that explicitly represents both haplotypes covering a genomic window. With $S$ denoting the number of windows of $M$ markers, consider a directed probabilistic graphical model (Figure 1) consisting of:

- $S$ hidden states, $y_{1: S}=\left(y_{1}, y_{2}, \ldots, y_{S}\right)$, where $y_{t}=\left(y_{t}^{0}, y_{t}^{1}\right) \in\{1, \ldots, K\}^{2}$ represents the true population labels of haplotypes 0 and 1 within window $t$;

- $S$ observed states, $x_{1: S}=\left(x_{1}, x_{2}, \ldots, x_{S}\right)$, where $x_{t}=\left(x_{t}^{0}, y_{t}^{1}\right) \in\{1, \ldots, N\}^{2}$ represents the observed population labels for the $t$-th pair of haplotype windows (i.e., the output from the local classifier); and

- $S-1$ hidden switch indicators, $s_{2: S}=\left(s_{2}, s_{3}, \ldots, s_{S}\right)$, where $s_{t} \in\{0,1\}$ denotes whether a phasing switch error has occurred between windows $t-1$ and $t$.

Note that we implicitly assume that phasing switch errors occur only at the boundaries between windows.

We model the joint probability of $y_{1: S}, x_{1: S}$, and $s_{2: S}$ as:

$$
P\left(y_{1: S}, x_{1: S}, s_{2: S}\right)=P\left(y_{1}\right) P\left(x_{1} \mid y_{1}\right) \prod_{t=2}^{S} P\left(s_{t}\right) P\left(y_{t} \mid y_{t-1}, s_{t}\right) P\left(x_{t} \mid x_{t-1}, y_{t-1}, y_{t}, s_{t}\right) .
$$




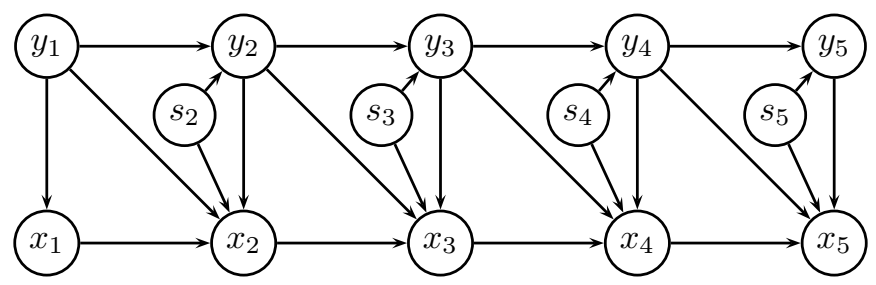

Figure 1: Graphical model of the error-correction module for sequence of length $S=5$.

We parameterize our model with $2\left(K^{2}+K\right)+1$ parameters:

- $\left\{\mu_{y}\right\}_{1: K}$, the prior distribution of hidden states following a recombination event;

- $\left\{\mu_{x \mid y}\right\}_{(1: K)^{2}}$, the prior distribution of emissions, conditional on hidden states;

- $\sigma$, the prior probability that a phasing switch error occurs between two consecutive windows;

- $\left\{\theta_{y}\right\}_{1: K}$, the prior probabilities of recombination between two consecutive windows, when the first has hidden state $y$; and

- $\left\{\varepsilon_{y, x}\right\}_{(1: K)^{2}}$, the prior probabilities of observed-state label resets between two consecutive windows, when the first has observed state $x$ and both have hidden state $y$.

We express each component of the joint probability expression (4) in terms of these parameters:

1. Initial hidden-state distribution. We assume that the population assignments for each of the two haplotypes is sampled independently from the stationary distribution of hidden states, $\pi$ :

$$
P\left(y_{1}\right)=\prod_{h \in\{0,1\}} \pi_{y_{1}^{h}}
$$

where

$$
\pi_{i}=\frac{\mu_{i} / \theta_{i}}{\sum_{j=1}^{K} \mu_{j} / \theta_{j}}
$$

We note that, in the original version of Ancestry Composition (Durand et al., 2014), the prior probability of recombination was a scalar, $\theta$, constant across hidden states. When this was the case, the stationary distribution of hidden states, $\boldsymbol{\pi}$, was equal to the prior distribution of hidden states, $\boldsymbol{\mu}$.

2. Initial emission distribution. The initial emissions for each haplotype are sampled independently from the prior distribution for emissions:

$$
P\left(x_{1} \mid y_{1}\right)=\prod_{h \in\{0,1\}} \mu_{x_{1}^{h} \mid y_{1}^{h}} .
$$

3. Switch error model. We assume that switch errors occur with constant probability $\sigma$ between each pair of states:

$$
P\left(s_{t}\right)=\sigma^{s_{t}}(1-\sigma)^{1-s_{t}} .
$$

4. Transition probability model. For each haplotype, a recombination occurs from hidden state $y$ with probability $\theta_{y}$, and for each recombination, we draw a new hidden population label from the prior distribution for hidden states. Thus,

$$
\begin{aligned}
P\left(y_{t} \mid y_{t-1}, s_{t}\right) & =\prod_{h \in\{0,1\}} P\left(y_{t}^{h} \mid y_{t-1}^{h \oplus s_{t}}\right) \\
& =\prod_{h \in\{0,1\}} f\left(y_{t-1}^{h \oplus s_{t}}, y_{t}^{h}\right)
\end{aligned}
$$


where

$$
f\left(y^{\prime}, y\right)=\theta_{y^{\prime}} \mu_{y}+\left(1-\theta_{y^{\prime}}\right) \mathbb{1}\left\{y^{\prime}=y\right\}
$$

5. Emission probability model. In order to accommodate correlated errors in local ancestry classifications, we designed the APHMM's emission model to be autoregressive: given no change in hidden state, the observed states are correlated. In our testing, this autoregressivity increased posterior decoding accuracy without any apparent performance decline.

As with the transition probability model, we treat each haplotype independently in the emission probability model. Consider two consecutive hidden states, $y_{t-1}$ and $y_{t}$. If they are unequal (i.e., a true ancestry switch has occurred), then an observed-state label reset necessarily occurs, and the emission at window $t, x_{t}$, is drawn from the prior distribution for emissions, $\left\{\mu_{x \mid y_{t}}\right\}$. If a true ancestry switch has not occurred (i.e., $y_{t-1}=y_{t} \equiv y$ ), an observed-state label reset occurs with probability $\varepsilon_{y, x_{t-1}}$ :

$$
P\left(x_{t} \mid x_{t-1}, y_{t-1}, y_{t}, s_{t}\right)=\prod_{h \in\{0,1\}} g\left(x_{t-1}^{h \oplus s_{t}}, x_{t}^{h}, y_{t-1}^{h \oplus s_{t}}, y_{t}^{h}\right)
$$

where

$$
g\left(x^{\prime}, x, y^{\prime}, y\right)= \begin{cases}\mu_{x \mid y} & \text { if } y^{\prime} \neq y \\ \varepsilon_{y, x^{\prime}} \mu_{x \mid y}+\left(1-\varepsilon_{y, x^{\prime}}\right) \mathbb{1}\left\{x=x^{\prime}\right\} & \text { if } y^{\prime}=y\end{cases}
$$

We estimate these model parameters using the expectation-maximization (EM) algorithm (Dempster et al., 1977). Posterior probabilities for each window are estimated using the forward and backward algorithms for hidden Markov models. Using dynamic programming techniques, the complexity of the posterior decoding step is $O\left(S K^{2}\right)$, where $S$ is the number of windows to decode and $K$ is the number of populations.

\subsection{Posterior aggregation for hierarchical classification}

Intracontinental local ancestry inference is a challenging problem, and it may not always be possible to confidently determine whether a segment derives from Scandinavia or the British Isles, either because we lack power or because the corresponding haplotypes occur at similar frequencies within the two populations. In such cases, it is often possible to confidently determine that the segment derives from a specific broader region (e.g., Northern Europe). Therefore, we have defined a four-level population hierarchy that groups populations within continents and regions (Figure 2). The $K$ leaves (i.e., terminal nodes) of our hierarchy correspond to the $K$ reference populations, and the highest level consists of a single root node representing the union of all populations. Broadly, the levels beneath the root correspond to continental-scale, regional-scale, and sub-regional-scale populations, respectively. Leaf nodes may occur at any of these levels; for example, Melanesia is placed immediately below the root, at the continent scale, and is not further subdivided.

For a given haplotype window, we sum the posterior probabilities from the leaves to the root of the tree, so that each node is assigned a probability equal to the sum of its children's probabilities, with the probability at the root always equal to one. We assign each haplotype window to the lowest node (i.e., the node closest to the leaves) at which the posterior probability exceeds a specified precision level, $t \in[0.5,1)$. In the worst case, no node other than the root has a posterior probability exceeding $t$. In this case, we do not classify the window. If assignment probabilities are well calibrated, this procedure ensures that the precision of the assignment is at least $t$. Therefore, we refer to $t$ as the "nominal precision threshold".

\subsection{Parameter estimation and model evaluation}

We used a stratified five-fold cross-validation approach to estimate parameters and evaluate models, maintaining similar representation among the 45 reference populations within each fold. For each fold, we estimated local-classifier parameters using a training set composed of the $\sim 80 \%$ of individuals assigned to the other folds. We then classified each window of each chromosome copy of each individual using the models trained with the individual held out, 
yielding hard-clustering vectors. To estimate emission parameters, including the autoregression transition matrix $\varepsilon$, we use a modified supervised EM algorithm applied to these hard-clustering vectors.

In the original implementation of Ancestry Composition (Durand et al., 2014), we estimated APHMM transition parameters using an unsupervised EM training procedure that relied on the natural admixture found in a broader set of 23andMe customers. Specifically, we estimated transition parameters for samples of $\sim 1000$ unrelated 23andMe customers from each of the following population groups: African-American, Ashkenazi Jewish, East Asian, European, Latino, Middle-Eastern, and South Asian. We term these groups "smoother training pools". For each individual to whom we applied Ancestry Composition prediction, we combined the predictions of each smoother training pool's model using Bayesian model averaging.

In the updated version of our algorithm, we estimate distinct APHMM transition parameter values for each individual to whom we apply Ancestry Composition prediction. To do so, we use the same EM algorithm that was used to estimate transition parameters for the smoother training pools, but, rather than aggregating expectations across $\sim 2000$ haplotypes for each chromosome, we aggregate across each individual's 23 pairs of hard-clustering vectors. To encourage convergence to sensible transition parameter values, we initialize transition-parameter optimization from the pretrained transition parameter sets of the smoother training pools. To determine which smoother training pool is to provide the initial values for transition parameter optimization, we use a multinomial Naive Bayes classifier trained on the hard-clustering assignments of all individuals in all smoother training pools. For each query individual to whom we apply Ancestry Composition, we initialize transition parameter values with those of the smoother training pool chosen by the Naive Bayes classifier when applied to the query individual's hard-clustering vectors. We find that this individualized transition-parameter optimization affords the error-correction module a great degree of flexibility and, in so doing, reduces bias and increases accuracy.

\section{Results}

We evaluated Ancestry Composition's classification performance using precision and recall measures computed via a five-fold stratified cross-validation experiment (see subsection 2.4). We estimated precision for population $k$ as the proportion of windows predicted to derive from population $k$ that actually do derive from population $k$, and we estimated recall for population $k$ as the proportion of windows truly deriving from population $k$ that were predicted to derive from population $k$.

Table 2 shows accuracy results at continental, regional, and sub-regional scales, as described in subsection 2.3. At each level of the population hierarchy, we estimated precision and recall for two precision thresholds: $t \in\{0.5,0.8\}$. Note that increasing the threshold increases precision at the expense of recall.

At the continental scale (i.e., for all non-leaf populations that are children of the root node), when $t=0.5$, precision exceeds $97 \%$ and recall exceeds $92 \%$. When $t=0.8$, precision is greater than $99 \%$ for all continents except Europe, which achieves a precision of $98.3 \%$, and recall drops slightly, with a minimum of $89.4 \%$ for West Asia and North Africa.

At the regional scale (i.e., considering the twelve non-continent non-leaf populations), precision and recall are uniformly less than or equal to the continent-scale parent populations, by definition. With nominal precision threshold $t=0.5$, precision remains fairly high, with median of $95.2 \%$, and it exceeds $90 \%$ for all but North Asia and Northwest Asia. Recall for $t=0.5$ is also relatively good at this scale, with median of $94.5 \%$. It exceeds $90 \%$ for eight of twelve regions and exceeds $80 \%$ for all. At nominal precision threshold $t=0.8$, precision has a median of $97.5 \%$ and is greater than $90 \%$ for all regions except North Asia and Northwest Asia. Recall decreases slightly but still remains above $85 \%$ for most populations.

At the leaf level, many populations continue to have good precision and recall metrics. Seven of 45 leaf populations (namely, Ethiopia \& Eritrea, Congo, Japan, Korea, China, Gujarati Subgroup, and Ashkenazi) achieve precision and recall greater than $95 \%$ for both nominal precision thresholds, and 17 of 45 leaf populations achieve precision and recall greater than $90 \%$ for both precision thresholds. At nominal precision threshold $t=0.5$, the median precision is $96.1 \%$ for all leaf-level populations, with $35 / 45$ leaf-level populations having precision at least $90 \%$ and $41 / 45$ populations having precision at least $80 \%$. Recall at $t=0.5$ has median $91.1 \%$, with $26 / 45$ leaf-level populations 
exceeding $90 \%$ and $38 / 45$ populations exceeding $80 \%$. As at the continental and regional scales, precision increases slightly and recall decreases slightly with nominal precision threshold $t=0.8$, as compared to $t=0.5$.

\section{Discussion}

We have developed a two-stage pipeline for ancestry deconvolution, Ancestry Composition. This modular approach makes our method flexible, robust, and easy to update. Ancestry Composition achieves high precision for closely related populations, as demonstrated by our cross-validation experiments, and it outputs probabilistic assignments, which enable confidence-threshold tuning and hierarchical classification. Unlike many previous approaches that can only distinguish between a few well-differentiated populations, Ancestry Composition can be trained to differentiate a large number of closely related populations.

The ultimate goal of ancestry deconvolution is to estimate $P(Y \mid X)$, the distribution of unobserved ancestry states $Y$, given observed haplotypes $X$. Generative approaches, such as HMMs, achieve this by first estimating the joint distribution $P(X, Y)$ and then conditioning on the observed data, $X$. In contrast, discriminative approaches directly model the conditional distribution $P(Y \mid X)$.

Discriminative approaches to classification often outperform generative methods (Lafferty et al., 2001), and the additional complexity required to fully model $P(X, Y)$ tends to limit generative ancestry deconvolution methods to just a few ancestral populations. In addition, discriminative approaches are typically more robust to model misspecification, precisely because they do not attempt to fully model the joint distribution of haplotypes and their ancestries. In light of these advantages, our local classifier does not assume any particular demographic model underlying the admixture process. Rather, our SKSVM learns decision boundaries between the reference populations directly from the data.

Despite the advantages discriminative approaches offer, generative models are generally more flexible and permit expression of more complex dependencies between observations and hidden random variables. Therefore, Ancestry Composition adopts a mixed approach, as advocated in Jaakkola et al. (1999) and Ng and Jordan (2002), in which the output of a discriminative local classifier is input to a generative error-correction module implemented as an autoregressive pair hidden Markov model. The hidden-Markov-model framework provides a natural means by which to correct phasing switch errors and model dependencies between adjacent observations.

In contrast to our mixed approach, RFMix (Maples et al., 2013) is a purely discriminative approach for admixture deconvolution. It implements random forests as its local classifier and conditional random fields to reconcile adjacent chromosomal windows. Random forests have some advantage over SVMs; they are inherently multiclass classifiers, and they output probabilities rather than hardcalls. However, SVMs offer a direct way to plug in a kernel, which enables us to efficiently extract an enormous number of features from short chromosomal segments.

Other purely discriminative approaches have recently been developed. Kumar et al. (2020) have described an approach that employs boosted gradient trees to perform local ancestry inference much faster and with fewer computational resources than existing methods, while maintaining comparable accuracy. A similar method, using neural networks has also been described recently (Montserrat et al., 2020).

\section{Acknowledgments}

We thank Brian Naughton, Katarzyna Bryc, Nicholas Eriksson, Robert Bell, Ethan Jewett, Will Freyman, Kimberly McManus, and other members of the 23 andMe Ancestry R\&D team for insightful comments throughout the development of this project. We also thank the many 23 andMe customers who answered survey questions and allowed us to study their genomes; this work would not have been possible without them. 


\section{References}

S. Bercovici, J. M. Rodriguez, M. Elmore, and S. Batzoglou. Ancestry inference in complex admixtures via variablelength Markov chain linkage models. In Proceedings of the 16th Annual Conference on Research in Computational Molecular Biology (RECOMB 2012), pages 12-28, 2012.

(Referenced in: 1.)

Bernhard E Boser, Isabelle M Guyon, and Vladimir N Vapnik. A training algorithm for optimal margin classifiers. In Proceedings of the fifth annual workshop on computational learning theory, pages 144-152. ACM, 1992.

(Referenced in: 2.1.1.)

Abra Brisbin, Katarzyna Bryc, Jake Byrnes, Fouad Zakharia, Larsson Omberg, Jeremiah Degenhardt, Andrew Reynolds, Harry Ostrer, Jason G Mezey, and Carlos D Bustamante. PCAdmix: principal components-based assignment of ancestry along each chromosome in individuals with admixed ancestry from two or more populations. Human Biology, 84(4):343-364, 2012.

(Referenced in: 1 and 1.)

Clare Bycroft, Colin Freeman, Desislava Petkova, Gavin Band, Lloyd T. Elliott, Kevin Sharp, Allan Motyer, Damjan Vukcevic, Olivier Delaneau, Jared O'Connell, Adrian Cortes, Samantha Welsh, Alan Young, Mark Effingham, Gil McVean, Stephen Leslie, Naomi Allen, Peter Donnelly, and Jonathan Marchini. The UK Biobank resource with deep phenotyping and genomic data. Nature, 562(7726):203-209, October 2018.

(Referenced in: 1.)

Howard M Cann, Claudia de Toma, Lucien Cazes, Marie-Fernande Legrand, Valerie Morel, Laurence Piouffre, Julia Bodmer, Walter F Bodmer, Batsheva Bonne-Tamir, Anne Cambon-Thomsen, Zhu Chen, Jiayou Chu, Carlo Carcassi, Licinio Contu, Ruofu Du, Laurent Excoffier, G B Ferrara, Jonathan S Friedlaender, Helena Groot, David Gurwitz, Trefor Jenkins, Rene J Herrera, Xiaoyi Huang, Judith Kidd, Kenneth K Kidd, Andre Langaney, Alice A Lin, S Qasim Mehdi, Peter Parham, Alberto Piazza, Maria Pia Pistillo, Yaping Qian, Qunfang Shu, Jiujin Xu, S Zhu, James L Weber, Henry T Greely, Marcus W Feldman, Gilles Thomas, Jean Dausset, and L Luca Cavalli-Sforza. A human genome diversity cell line panel. Science, 296(5566):261-2, Apr 2002.

(Referenced in: 2.1.2.)

Nello Cristianini and John Shawe-Taylor. An Introduction to Support Vector Machines and Other Kernel-Based Learning Methods. Cambridge University Press, 2000.

(Referenced in: 2.1.1.)

A. P. Dempster, N. M. Laird, and D. B. Rubin. Maximum likelihood from incomplete data via the EM algorithm. Journal of the Royal Statistical Society, Series B, 39(1):1-38, 1977.

(Referenced in: 2.2.)

Alex Diaz-Papkovich, Luke Anderson-Trocmé, Chief Ben-Eghan, and Simon Gravel. UMAP reveals cryptic population structure and phenotype heterogeneity in large genomic cohorts. PLOS Genetics, 15(11):e1008432, Nov 2019. (Referenced in: 2.1.2.)

E. Y. Durand, C. B. Do, J. L. Mountain, and J. M. Macpherson. Ancestry Composition: A Novel, Efficient Pipeline for Ancestry Deconvolution. bioRxiv, page 010512, 2014. doi: 10.1101/010512.

(Referenced in: 1, 1, and 2.4.)

D. Falush, M. Stephens, and J. K. Pritchard. Inference of population structure using multilocus genotype data: linked loci and correlated allele frequencies. Genetics, 164(4):1567-1587, Aug 2003.

(Referenced in: 1.)

Garrett Hellenthal, George B. J. Busby, Gavin Band, James F. Wilson, Cristian Capelli, Daniel Falush, and Simon Myers. A genetic atlas of human admixture history. Science, 343(6172):747-751, February 2014.

(Referenced in: 1.)

Brenna M Henn, Lawrence Hon, J Michael Macpherson, Nick Eriksson, Serge Saxonov, Itsik Pe'er, and Joanna L Mountain. Cryptic distant relatives are common in both isolated and cosmopolitan genetic samples. PLOS One, 7 (4):e34267, 2012.

(Referenced in: 2.1.2.) 
Tommi Jaakkola, David Haussler, et al. Exploiting generative models in discriminative classifiers. Advances in Neural Information Processing Systems, pages 487-493, 1999.

(Referenced in: 4.)

Arvind Kumar, Daniel Mas Montserrat, Carlos Bustamante, and Alexander Ioannidis. XGMix: Local-Ancestry Inference with Stacked XGBoost. bioRxiv, page 2020.04.21.053876, April 2020.

(Referenced in: 1 and 4.)

John Lafferty, Andrew McCallum, and Fernando Pereira. Conditional random fields: Probabilistic models for segmenting and labeling sequence data. Proceedings of the 18th International Conference on Machine Learning (ICML2001), June 2001.

(Referenced in: 4.)

Po-Ru Loh, Petr Danecek, Pier Francesco Palamara, Christian Fuchsberger, Yakir A. Reshef, Hilary K. Finucane, Sebastian Schoenherr, Lukas Forer, Shane McCarthy, Goncalo R. Abecasis, Richard Durbin, and Alkes L. Price. Reference-based phasing using the Haplotype Reference Consortium panel. Nature Genetics, 48(11):1443-1448, November 2016.

(Referenced in: 1.)

Brian K Maples, Simon Gravel, Eimear E Kenny, and Carlos D Bustamante. RFMix: A discriminative modeling approach for rapid and robust local-ancestry inference. The American Journal of Human Genetics, 93(2):278-288, August 2013.

(Referenced in: 1, 1, and 4.)

Shane McCarthy, Sayantan Das, Warren Kretzschmar, Olivier Delaneau, Andrew R. Wood, Alexander Teumer, Hyun Min Kang, Christian Fuchsberger, Petr Danecek, Kevin Sharp, Yang Luo, Carlo Sidore, Alan Kwong, Nicholas Timpson, Seppo Koskinen, Scott Vrieze, Laura J. Scott, He Zhang, Anubha Mahajan, Jan Veldink, Ulrike Peters, Carlos Pato, Cornelia M. van Duijn, Christopher E. Gillies, Ilaria Gandin, Massimo Mezzavilla, Arthur Gilly, Massimiliano Cocca, Michela Traglia, Andrea Angius, Jeffrey Barrett, Dorret I. Boomsma, Kari Branham, Gerome Breen, Chad Brummet, Fabio Busonero, Harry Campbell, Andrew Chan, Sai Chen, Emily Chew, Francis S. Collins, Laura Corbin, George Davey Smith, George Dedoussis, Marcus Dorr, Aliki-Eleni Farmaki, Luigi Ferrucci, Lukas Forer, Ross M. Fraser, Stacey Gabriel, Shawn Levy, Leif Groop, Tabitha Harrison, Andrew Hattersley, Oddgeir L. Holmen, Kristian Hveem, Matthias Kretzler, James Lee, Matt McGue, Thomas Meitinger, David Melzer, Josine Min, Karen L. Mohlke, John Vincent, Matthias Nauck, Deborah Nickerson, Aarno Palotie, Michele Pato, Nicola Pirastu, Melvin McInnis, Brent Richards, Cinzia Sala, Veikko Salomaa, David Schlessinger, Sebastian Schoenheer, P Eline Slagboom, Kerrin Small, Timothy Spector, Dwight Stambolian, Marcus Tuke, Jaakko Tuomilehto, Leonard Van den Berg, Wouter Van Rheenen, Uwe Volker, Cisca Wijmenga, Daniela Toniolo, Eleftheria Zeggini, Paolo Gasparini, Matthew G. Sampson, James F. Wilson, Timothy Frayling, Paul de Bakker, Morris A. Swertz, Steven McCarroll, Charles Kooperberg, Annelot Dekker, David Altshuler, Cristen Willer, William Iacono, Samuli Ripatti, Nicole Soranzo, Klaudia Walter, Anand Swaroop, Francesco Cucca, Carl Anderson, Michael Boehnke, Mark I. McCarthy, Richard Durbin, Gonçalo Abecasis, and Jonathan Marchini. A reference panel of 64,976 haplotypes for genotype imputation. Nature Genetics, 48(10):1279-1283, October 2016.

(Referenced in: 1.)

Leland McInnes, John Healy, Nathaniel Saul, and Lukas Großberger. UMAP: Uniform manifold approximation projection. Journal of Open Source Software, 3(29):861, 2018.

(Referenced in: 2.1.2.)

Daniel Mas Montserrat, Carlos Bustamante, and Alexander Ioannidis. LAI-Net: Local-ancestry inference with neural networks. ICASSP 2020 - 2020 IEEE International Conference on Acoustics, Speech and Signal Processing (ICASSP), pages 1314-1318, May 2020.

(Referenced in: 1 and 4.)

A.Y. Ng and A Jordan. On discriminative vs. generative classifiers: A comparison of logistic regression and naive Bayes. Advances in neural information processing systems, 14:841, 2002.

(Referenced in: 4.)

John Novembre and Sohini Ramachandran. Perspectives on human population structure at the cusp of the sequencing 
era. Annual Review of Genomics and Human Genetics, 12(1):245-274, 2011.

(Referenced in: 1.)

Larsson Omberg, Jacqueline Salit, Neil Hackett, Jennifer Fuller, Rebecca Matthew, Lotfi Chouchane, Juan L Rodriguez-Flores, Carlos Bustamante, Ronald G Crystal, and Jason G Mezey. Inferring genome-wide patterns of admixture in qataris using fifty-five ancestral populations. BMC Genetics, 13(1):49, 2012.

(Referenced in: 1 and 1.)

B. Pasaniuc, S. Sankararaman, G. Kimmel, and E. Halperin. Inference of locus-specific ancestry in closely related populations. Bioinformatics, 25(12):i213-i221, June 2009.

(Referenced in: 1 and 1.)

Alkes L Price, Arti Tandon, Nick Patterson, Kathleen C Barnes, Nicholas Rafaels, Ingo Ruczinski, Terri H Beaty, Rasika Mathias, David Reich, and Simon Myers. Sensitive detection of chromosomal segments of distinct ancestry in admixed populations. PLOS Genetics, 5(6):e1000519, 2009.

(Referenced in: 1 and 1.)

Alkes L. Price, Noah A. Zaitlen, David Reich, and Nick Patterson. New approaches to population stratification in genome-wide association studies. Nature Reviews Genetics, 11(7):459-463, June 2010.

(Referenced in: 1.)

Gunnar Rätsch, Sören Sonnenburg, and Christin Schäfer. Learning interpretable SVMs for biological sequence classification. BMC Bioinformatics, 7(Suppl 1):S9, 2006.

(Referenced in: 2.1.1.)

Michael F. Seldin, Bogdan Pasaniuc, and Alkes L. Price. New approaches to disease mapping in admixed populations. Nature Reviews Genetics, 12(8):523-528, August 2011.

(Referenced in: 1.)

A. Sundquist, E. Fratkin, C. B. Do, and S. Batzoglou. Effect of genetic divergence in identifying ancestral origin using HAPAA. Genome Research, 18(4):676-682, Apr 2008.

(Referenced in: 1.)

H. Tang, J. Peng, P. Wang, and N. J. Risch. Estimation of individual admixture: analytical and study design considerations. Genetic Epidemiology, 28(4):289-301, May 2005.

(Referenced in: 1.)

Hua Tang, Marc Coram, Pei Wang, Xiaofeng Zhu, and Neil Risch. Reconstructing genetic ancestry blocks in admixed individuals. The American Journal of Human Genetics, 79(1):1-12, 2006.

(Referenced in: 1.)

The 1000 Genomes Project Consortium. A global reference for human genetic variation. Nature, 526(7571):68-74, 2015 .

(Referenced in: 2.1.2.)

Vladimir N Vapnik. Statistical Learning Theory. Wiley, 1998. ISBN 0471030031.

(Referenced in: 2.1.1.) 
bioRxiv preprint doi: https://doi.org/10.1101/2021.01.19.427308; this version posted January 20, 2021. The copyright holder for this preprint (which was not certified by peer review) is the author/funder. All rights reserved. No reuse allowed without permission.

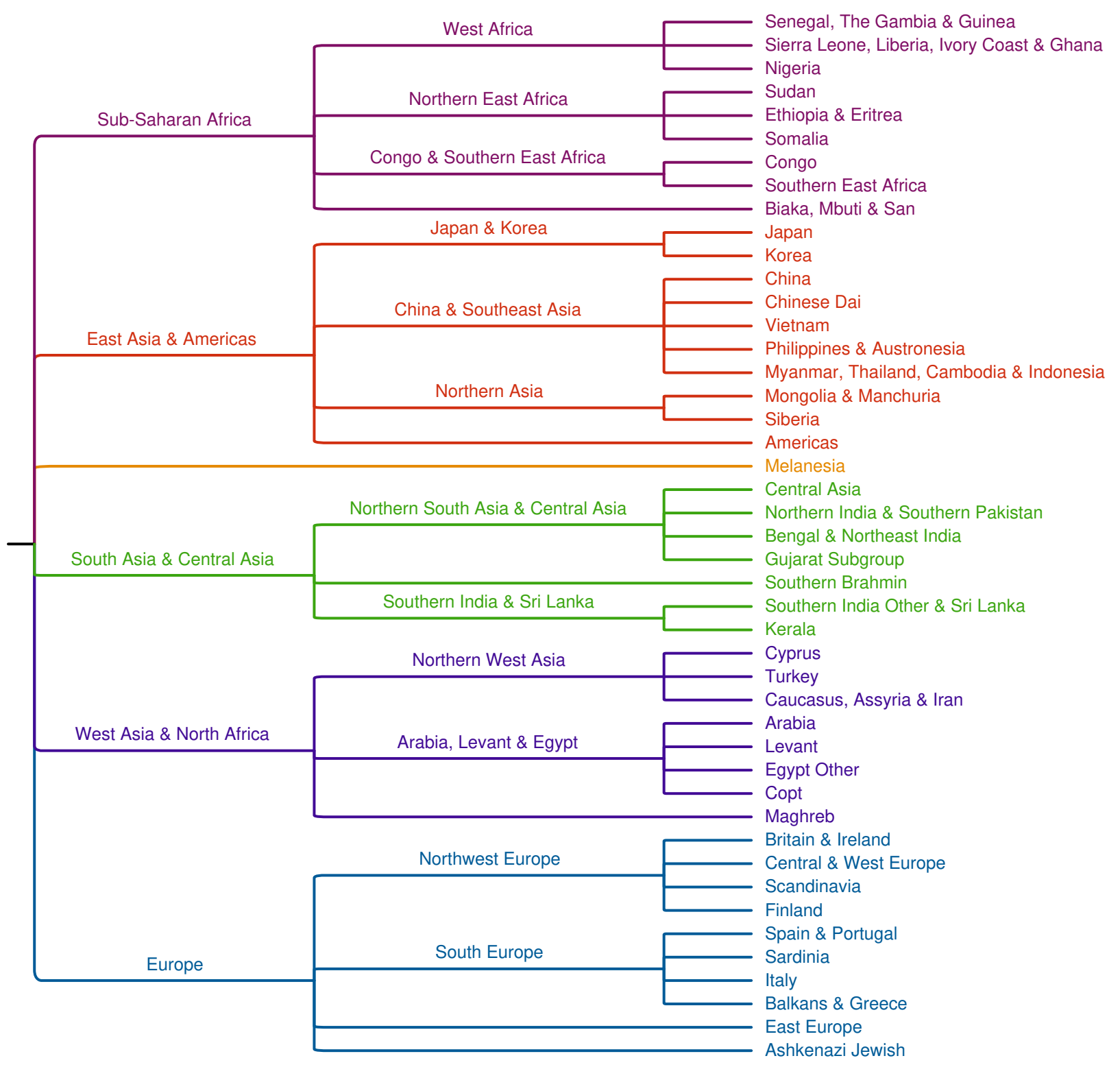

Figure 2: Population hierarchy with 45 reference populations (leaves). Colors reflect the six continental groupings at the highest level of the hierarchy. 
Table 1: Reference population sample composition. For Mongolia \& Manchuria, the count of individuals from the 23 andMe database is between one and five, with the totals in the margins reflecting a count of five in that cell.

\begin{tabular}{lrrr} 
Population & 23andMe & Public & Total \\
\hline Americas & 12 & 62 & 74 \\
Arabia & 257 & 0 & 257 \\
Ashkenazi Jewish & 1007 & 0 & 1007 \\
Balkans \& Greece & 614 & 0 & 614 \\
Bengal \& Northeast India & 166 & 80 & 246 \\
Biaka, Mbuti \& San & 0 & 41 & 41 \\
Britain \& Ireland & 935 & 79 & 1014 \\
Caucasus, Assyria \& Iran & 400 & 0 & 400 \\
Central \& West Europe & 936 & 21 & 957 \\
Central Asia & 113 & 24 & 137 \\
China & 544 & 251 & 795 \\
Chinese Dai & 0 & 82 & 82 \\
Congo & 597 & 0 & 597 \\
Copt & 120 & 0 & 120 \\
Cyprus & 158 & 0 & 158 \\
East Europe & 761 & 24 & 785 \\
Egypt Other & 185 & 0 & 185 \\
Ethiopia \& Eritrea & 171 & 0 & 171 \\
Finland & 279 & 85 & 364 \\
Gujarat Subgroup & 85 & 67 & 152 \\
Italy & 482 & 114 & 596 \\
Japan & 346 & 128 & 474 \\
Kerala & 191 & 0 & 191 \\
Korea & 341 & 0 & 341 \\
Levant & 348 & 52 & 400 \\
Maghreb & 307 & 25 & 332 \\
Melanesia & 0 & 29 & 29 \\
Mongolia \& Manchuria & 114 & 83 \\
Myanmar, Thailand, Cambodia \& Indonesia & 69 & 2027 & $\sim 14,445$ \\
Nigeria & 18 & $\sim 23$ \\
Northern India \& Southern Pakistan & 8 & 77 \\
Philippines \& Austronesia & 54 & 226 & 280 \\
Sardinia & 254 & 46 & 300 \\
Scandinavia & 164 & 0 & 164 \\
Senegal, The Gambia \& Guinea & 0 & 25 & 25 \\
Siberia & 631 & 0 & 631 \\
Sierra Leone, Liberia, Ivory Coast \& Ghana & 23 & 135 & 158 \\
Somalia & 0 & 22 & 22 \\
Southern Brahmin & 196 & 85 & 281 \\
Southern East Africa & 150 & 0 & 150 \\
Southern India Other \& Sri Lanka & 292 & 8 & 300 \\
Spain \& Portugal & 13 & 124 \\
Sudan & 09 & 289 \\
Turkey & 357 \\
Vietnam & 0 & 189 \\
Total & 09 \\
\hline
\end{tabular}


bioRxiv preprint doi: https://doi.org/10.1101/2021.01.19.427308; this version posted January 20, 2021. The copyright holder for this preprint (which was not certified by peer review) is the author/funder. All rights reserved. No reuse allowed without permission.

Table 2: Precision and recall (\%) for all populations in the population hierarchy.

\begin{tabular}{|c|c|c|c|c|}
\hline & $\begin{array}{r}\text { Precision } \\
t=0.5\end{array}$ & $\begin{array}{r}\text { Recall } \\
t=0.5\end{array}$ & $\begin{array}{r}\text { Precision } \\
t=0.8\end{array}$ & $\begin{array}{r}\text { Recall } \\
t=0.8\end{array}$ \\
\hline Sub-Saharan Africa & 99.0 & 98.6 & 99.2 & 98.2 \\
\hline West Africa & 98.4 & 98.9 & 99.0 & 98.1 \\
\hline Senegal, The Gambia \& Guinea & 94.5 & 96.0 & 97.0 & 91.3 \\
\hline Sierra Leone, Liberia, Ivory Coast \& Ghana & 96.6 & 87.4 & 98.6 & 74.8 \\
\hline Nigeria & 91.6 & 98.9 & 96.7 & 95.3 \\
\hline Northern East Africa & 97.5 & 92.7 & 98.2 & 91.1 \\
\hline Sudan & 95.0 & 83.8 & 96.3 & 79.9 \\
\hline Ethiopia \& Eritrea & 93.9 & 97.8 & 95.8 & 97.1 \\
\hline Somalia & 99.0 & 91.6 & 99.3 & 90.4 \\
\hline Congo \& Southern East Africa & 97.4 & 99.4 & 98.5 & 98.2 \\
\hline Congo & 98.1 & 99.3 & 99.2 & 97.6 \\
\hline Southern East Africa & 93.5 & 97.1 & 96.6 & 92.7 \\
\hline Biaka, Mbuti \& San & 98.5 & 86.3 & 99.2 & 80.2 \\
\hline East Asia \& Americas & 98.7 & 99.5 & 99.0 & 99.3 \\
\hline Japan \& Korea & 98.6 & 99.9 & 99.0 & 99.9 \\
\hline Japan & 99.7 & 99.2 & 99.7 & 99.0 \\
\hline Korea & 96.0 & 99.6 & 97.0 & 99.5 \\
\hline China \& Southeast Asia & 99.6 & 98.0 & 99.7 & 97.0 \\
\hline China & 96.7 & 94.3 & 97.5 & 91.4 \\
\hline Chinese Dai & 81.6 & 98.2 & 87.4 & 97.3 \\
\hline Vietnam & 94.4 & 98.0 & 96.7 & 97.2 \\
\hline Philippines \& Austronesia & 93.8 & 89.6 & 94.7 & 86.5 \\
\hline Myanmar, Thailand, Cambodia \& Indonesia & 94.9 & 66.5 & 95.8 & 57.9 \\
\hline Northern Asia & 56.0 & 95.0 & 67.3 & 92.3 \\
\hline Mongolia \& Manchuria & 38.1 & 91.6 & 50.7 & 86.1 \\
\hline Siberia & 91.9 & 95.1 & 95.3 & 92.4 \\
\hline Americas & 98.8 & 94.1 & 99.3 & 90.3 \\
\hline Melanesia & 98.8 & 98.3 & 99.2 & 96.9 \\
\hline South Asia \& Central Asia & 98.5 & 96.9 & 98.9 & 95.9 \\
\hline Northern South Asia \& Central Asia & 94.3 & 91.6 & 95.8 & 88.7 \\
\hline Central Asia & 86.1 & 49.4 & 88.5 & 37.4 \\
\hline Northern India \& Southern Pakistan & 82.3 & 87.8 & 85.5 & 83.0 \\
\hline Bengal \& Northeast India & 91.6 & 94.7 & 94.6 & 92.6 \\
\hline Gujarat Subgroup & 98.2 & 97.7 & 98.7 & 96.8 \\
\hline Southern Brahmin & 93.3 & 83.9 & 95.0 & 80.1 \\
\hline Southern India \& Sri Lanka & 89.8 & 94.2 & 92.3 & 91.9 \\
\hline Southern India Other \& Sri Lanka & 78.1 & 93.3 & 82.1 & 90.6 \\
\hline Kerala & 92.8 & 75.5 & 94.6 & 73.5 \\
\hline West Asia \& North Africa & 95.8 & 95.3 & 97.1 & 93.4 \\
\hline Northern West Asia & 83.7 & 92.0 & 87.7 & 89.0 \\
\hline Cyprus & 94.7 & 93.3 & 97.0 & 90.2 \\
\hline Turkey & 86.2 & 67.6 & 91.8 & 56.2 \\
\hline Caucasus, Assyria \& Iran & 67.1 & 92.9 & 74.6 & 88.8 \\
\hline Arabia, Levant \& Egypt & 94.3 & 85.6 & 95.8 & 81.8 \\
\hline Arabia & 88.2 & 70.3 & 91.2 & 66.1 \\
\hline Levant & 93.8 & 70.5 & 95.7 & 63.4 \\
\hline Egypt Other & 74.1 & 90.2 & 82.1 & 86.4 \\
\hline Copt & 92.4 & 95.3 & 95.1 & 93.7 \\
\hline Maghreb & 97.4 & 89.9 & 98.4 & 86.7 \\
\hline Europe & 98.7 & 99.0 & 99.0 & 98.4 \\
\hline Northwest Europe & 96.1 & 96.3 & 97.2 & 94.0 \\
\hline Britain \& Ireland & 95.4 & 88.5 & 98.2 & 75.4 \\
\hline Central \& West Europe & 79.7 & 81.3 & 87.1 & 64.4 \\
\hline Scandinavia & 90.8 & 90.7 & 94.9 & 81.9 \\
\hline Finland & 93.5 & 95.3 & 95.3 & 93.3 \\
\hline South Europe & 91.1 & 89.7 & 94.0 & 85.2 \\
\hline Spain \& Portugal & 90.0 & 96.5 & 94.1 & 94.0 \\
\hline Sardinia & 88.1 & 95.2 & 92.3 & 92.3 \\
\hline Italy & 87.8 & 86.1 & 92.1 & 79.1 \\
\hline Balkans \& Greece & 89.5 & 81.0 & 92.5 & 75.0 \\
\hline East Europe & 85.9 & 88.8 & 89.4 & 82.6 \\
\hline Ashkenazi Jewish & 99.3 & 98.6 & 99.4 & 98.1 \\
\hline
\end{tabular}

